
la Grande Guerre

\title{
L'empreinte américaine durant la Grande Guerre : persistances des vestiges américains en France (1917 à 1919)
}

Alexandre Coulaud, Manon Mariette et Robin Perarnau

\section{OpenEdition}

\section{Journals}

Édition électronique

URL : https://journals.openedition.org/geohist/672

DOI : 10.4000/geohist. 672

ISSN : 2264-2617

Éditeur

Association française de la Revue de géographie historique

Référence électronique

Alexandre Coulaud, Manon Mariette et Robin Perarnau, « L'empreinte américaine durant la Grande

Guerre : persistances des vestiges américains en France (1917 à 1919) », Revue de géographie

historique [En ligne], 14-15 | 2019, mis en ligne le 28 mai 2018, consulté le 12 juin 2021. URL : http:// journals.openedition.org/geohist/672 ; DOI : https://doi.org/10.4000/geohist.672

Ce document a été généré automatiquement le 12 juin 2021.

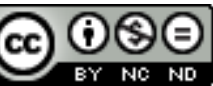

$\mathrm{Ce}(\mathrm{tte})$ œuvre est mise à disposition selon les termes de la Licence Creative Commons Attribution -

Pas d'Utilisation Commerciale - Pas de Modification 4.0 International. 


\title{
L'empreinte américaine durant la Grande Guerre : persistances des vestiges américains en France (1917 à 1919)
}

\author{
Alexandre Coulaud, Manon Mariette et Robin Perarnau
}

\section{Introduction}

1 En 1917, l'alignement des États-Unis aux forces de la Triple Entente entraîne un des plus importants déploiements logistiques de l'Histoire : 2 millions d'hommes vont traverser l'Atlantique, emportant avec eux de la plus petite planche de bois à la locomotive. De cette opération extraordinaire, il ne subsiste presque rien dans la mémoire collective. Cependant, les travaux de recherches menés par les archéologues de l'Inrap et de l'Afrac ont permis de faire évoluer notre perception de la participation américaine dans le premier conflit mondial. Au-delà des témoignages et des archives, la réalité archéologique laisse entrevoir un formidable déploiement militaire à l'instar des opérations militaires actuelles. L'inventaire effectué en 2016 recense près de 600 infrastructures construites entre 1917 et 1919, réparties sur 200 sites à travers tout le territoire français (Perarnau, 2016). Ces nouvelles installations (hôpitaux, usines, laboratoires, réseaux hydrauliques) contribuent au développement industriel et humain du territoire français ainsi qu'à l'effort de guerre.

\section{Les américains dans la Grande Guerre}

\section{A. 1917 : l'entrée des États-Unis dans la Grande Guerre}

En avril 1917, les États-Unis sortent de leur neutralité sous l'impulsion du président Woodrow Wilson. Le 14 mai, le maréchal Joffre et le secrétaire à la Guerre Newton D. 
Baker signent un accord stipulant les conditions de l'intervention américaine en Europe aux côtés des Alliés. Celui-ci prévoit l'envoi d'un corps expéditionnaire (American Expeditionnary Forcesou A.E.F.) dont l'armement et les munitions seront fournis par la France en contrepartie de l'envoi des matières premières nécessaires à leur fabrication.

3 À terme, les États-Unis envisagent le débarquement d'environ 4 millions d'hommes en France, sous le commandement du Général Pershing. Toutefois, au début du mois de juin 1917, cette force expéditionnaire ne se résume qu'à une avant-garde d'environ 20 000 hommes (principalement des soldats de l'United States Marines Corps, les plus expérimentés). La formation des premières troupes américaines débute au mois de juillet suivant, sous commandement français. Celui-ci note une volonté de fer chez les nouveaux arrivants mais également leur cruelle inexpérience et leur matériel inadapté aux conditions de la guerre moderne (Miquel, 1983).

4 À la signature de l'Armistice, bien que 2 millions de soldats américains se trouvent alors sur le sol français, seuls 400000 d'entre eux sont armés et aptes au combat, une part importante du contingent étant employée au soutien logistique de l'armée. Par ailleurs, avec l'appui de la France qui fournit l'ensemble de l'armement lourd (avions, chars et canons notamment), l'armée américaine devient, au terme du conflit, l'armée la plus mécanisée au monde (Miquel, 1983).

\section{B. La logistique de l'American Expeditionnary Forces en France}

La volonté du président Wilson d'une armée indépendante permet au corps expéditionnaire d'être déployé sur un secteur spécifique du front, au nord-est de la France et plus particulièrement en Lorraine et en Champagne (Coulaud et Tisserand, 2016a ; 2016b). L'acheminement, par rail, des soldats et du matériel en provenance des ports de débarquement vers le front constitue alors le principal problème logistique des troupes américaines. En effet, l'engorgement des réseaux ferrés français oblige les ingénieurs américains à construire de nouvelles infrastructures ferroviaires dès juin 1917. Deux lignes principales pourvues de nouvelles gares régulatrices sont alors mises en place pour l'acheminement des hommes et du matériel. Des contournements sont également implantés afin d'éviter les importants centres ferroviaires déjà saturés par les forces alliées, à l'image du cut-off de Nevers (Fig. 1).

\section{Document 1 : Lecut-offde Nevers (Nièvre).}

A : Persistances dans le paysage actuel de la voie ferrée (1) et du pont (2) (IGN, DAO A. Coulaud, Inrap). B : Exemples de pieux de bois composant les chevalets du pont conservés dans le lit de la Loire (M. Mariette, Afrac). C : Vue générale du pont ferroviaire durant le conflit (U.S. Signal Corps) 


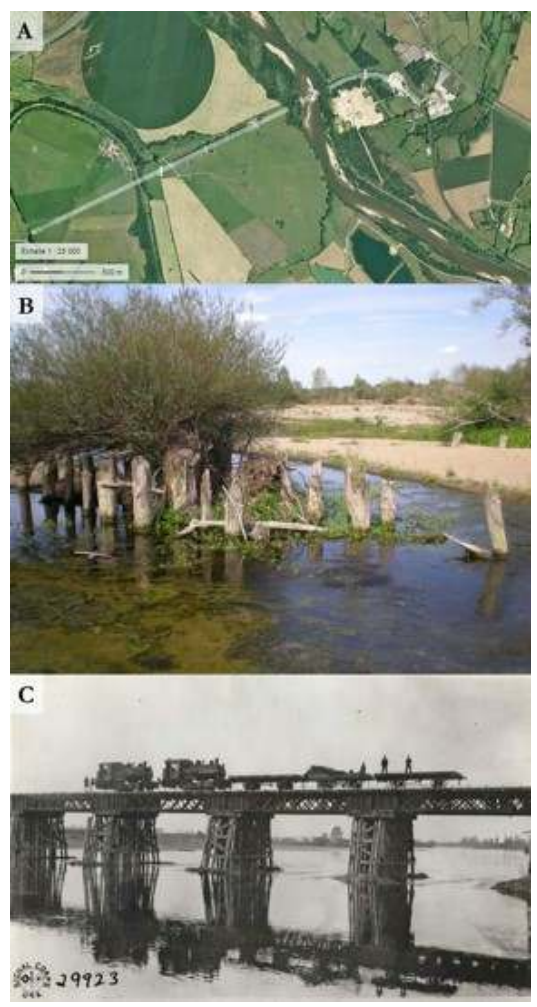

6 Ces lignes d'approvisionnement, tout comme les zones de débarquement, sont jalonnées par de très nombreuses infrastructures : gares régulatrices, dépôts logistiques et de munitions, ateliers d'assemblage et de réparation, complexes hospitaliers, hôpitaux vétérinaires ainsi que camps de transit, de repos et d'entrainement. À ce jour, l'inventaire fait état d'approximativement 600 implantations réparties sur près de 200 localités à travers tout le territoire français (Perarnau, 2016).

\section{Détecter les vestiges d'une occupation événementielle}

\section{A.Systèmes d'Informations Géographiqueset imagerie satellitaire au service de l'archéologie}

7 L'étude des photographies anciennes permet de reconstituer les plans de camps américains, parfois disparus des archives. Ainsi à Beaune (Côte-d'Or) et à Mesves-Bulcy (Nièvre), aucun plan complet de ces hôpitaux ne subsiste aujourd'hui, que ce soit dans les archives américaines ou dans les fonds français. Il faut donc nécessairement utiliser la photographie aérienne ancienne pour pouvoir les reconstituer, au moins partiellement. Les photographies de 1940 et 1950 ont ainsi permis d'établir un nouveau plan du centre hospitalier de Beaune (Perarnau, 2016). Les vestiges, encore visibles sur ces clichés, ont pu être identifiés. Le traitement des données photographiques a ainsi permis de reconstituer une véritable cartographie des lieux.

8 La mise en relation du Système d'Information Géographique (SIG) avec les données archivistiques permet d'identifier la fonctionnalité des vestiges par leur position relative. Ainsi, à Mars-sur-Allier, chaque structure repérée en prospection est 
désormais bien catégorisée grâce à son identification sur le plan-type d'une Base Hospital(Mariette, 2017) (Fig. 2).

Document 2:Organisation générale de l'Hospital Center de Mars-sur-Allier (M. Mariette, Afrac).

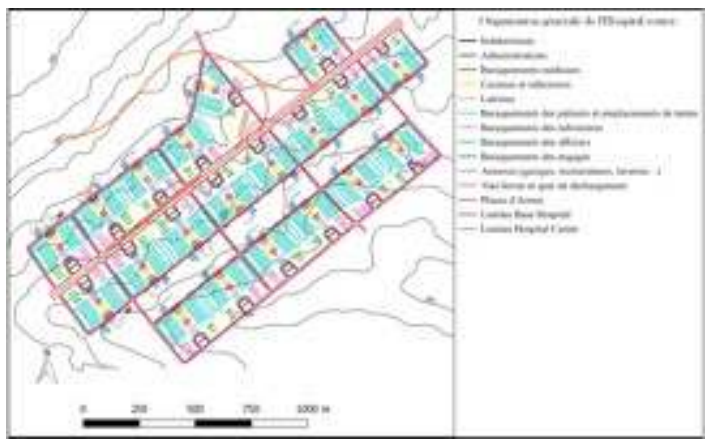

10 L'utilisation d'un SIG permet d'avoir une vision d'ensemble des vestiges repérés par prospection ou lors d'opérations archéologiques. Il est ainsi possible d'établir un lien entre différentes structures distantes de plusieurs dizaines de mètres. Dans le cas du centre hospitalier de Mars-sur-Allier, un nombre important d'infrastructures hydrauliques a été mis en évidence par prospection pédestre. Les archives ne mentionnant que très peu ces éléments, leur identification s'est avérée difficile. Néanmoins, l'étude de la répartition des vestiges indique que ces différentes structures appartiennent à un même ensemble. En étudiant leur place dans cet ensemble, leur fonction a ainsi pu être déterminée : il s'agit de réservoirs semi-enterrés, de canalisations, et d'installations plus complexes. Une reconstitution complète du système hydraulique du site de l'hôpital a ainsi pu être élaborée. Ce dernier comprend un système d'adduction et d'évacuation des eaux usées, très perfectionné pour l'époque. Ces infrastructures jouent un rôle sanitaire très important dans le fonctionnement de ces hôpitaux (Mariette, 2017).

\section{B. La prospection géophysique sur le centre hospitalier américain de Mars-sur-Allier}

11 L'étude géophysique peut, elle aussi, révéler certaines traces d'activités anthropiques effacées par le temps. Ainsi, à Mars-sur-Allier, l'étude menée en 2018 montre la présence d'un ancien cimetière et de baraquements liés à l'activité de l'hôpital (Mariette, 2018).

Des prospections magnétiques ont été réalisées sur le secteur du cimetière. Les images obtenues mettent en évidence des anomalies formant un maillage régulier et symétrique. Celui-ci correspond probablement à la fossilisation des allées de passages entre les tombes de la nécropole. Cette interprétation résulte de plusieurs observations. D'abord, les anomalies linéaires formant le maillage sont magnétiquement positives. Elles peuvent correspondre à un tassement des argiles (présentes naturellement sur cette zone) ou bien à un aménagement des allées. Dans les deux cas, il s'agit ici de traces d'une occupation anthropique ancienne.

De plus, les nombreuses anomalies négatives situées entre les mailles du quadrillage peuvent être identifiées comme les vestiges des amas de pierre calcaire disposés en 
surface afin de marquer les tombes. Ces éléments ne sont plus observables dans le paysage actuel.

À partir de ces informations, des gabarits de cercueils type ont été projetés sur le maillage afin de déterminer la possibilité d'inhumer 438 personnes, comme l'évoquent les archives (Fig. 3).

Document 3 :Hypothèse d'interprétation des résultats des prospections magnétiques sur le secteur du cimetière de l'hospital centerde Mars-sur-Allier, Nièvre (DAO : M. Mariette, Afrac).

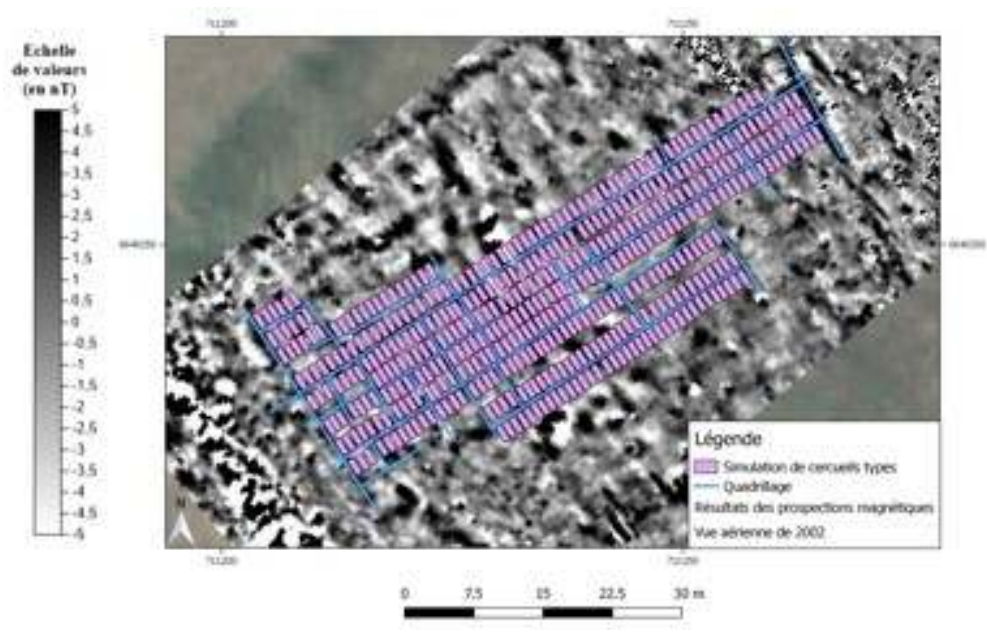

En s'appuyant sur les résultats des fouilles archéologiques de cimetières provisoires contemporains, l'hypothèse d'un écart de $40 \mathrm{~cm}$ entre les fosses individuelles semble pertinente. Les 438 cercueils forment alors un ensemble régulier et cohérent qui s'associe parfaitement aux espaces de circulation. Il est néanmoins possible d'identifier deux secteurs distincts : à l'ouest, les allées délimitent des aires quadrangulaires de 3 à $4 \mathrm{~m}$ de côté tandis que le côté est du complexe est caractérisé par de grandes anomalies linéaires se prolongeant sur plusieurs dizaines de mètres. Plusieurs hypothèses peuvent être émises pour identifier les raisons d'une telle organisation. Celle-ci peut refléter l'existence de différentes phases d'occupation du cimetière, rythmées par la vie de l'hôpital (Mariette, 2018). Il est ainsi possible que plusieurs dynamiques d'occupation liées au nombre de décès se soient succédées. Ainsi, les périodes de faible mortalité permettent aux autorités d'inhumer progressivement les morts de manière individuelle tandis que les périodes de grandes offensives ou d'épidémies impliquent le recours à des tranchées d'inhumation comme sépultures multiples.

L'existence d'une hiérarchisation des sépultures est également envisageable. Considérant la grande distance séparant le centre hospitalier de Mars-sur-Allier du front, il est possible que les mises en terre aient été faites de manière organisée et réfléchie. Il se pourrait donc qu'il y ait une distinction entre les sépultures destinées aux membres du personnel du site, correspondant probablement à des tombes individuelles situées à l'ouest, et celles dédiées aux patients, plutôt inhumés collectivement dans les tranchées à l'est du cimetière, le long de grandes allées de circulation.

Bien que certains sites de la Grande Guerre situés à l'arrière du front aient disparu du paysage actuel et de la mémoire collective, les fondations des vestiges maçonnés sont souvent préservées dans le sol. Grâce à la géophysique, ces structures peuvent être identifiées et cartographiées précisément, sans processus intrusif. 
À Mars-sur-Allier une partie de la zone de baraquements de la base hospital14 (unité hospitalière indépendante) du complexe hospitalier a fait l'objet de ce type d'études (Mariette, 2018). Les données obtenues permettent de reconstituer le plan des bâtiments, pourtant invisibles au sol. La confrontation de ces résultats aux données archivistiques apporte de réelles clés de lecture pour la détermination de la fonction de chaque baraque, chaque type de source complétant l'autre. Ainsi, les données des prospections géophysiques ont permis de nuancer les données archivistiques en mettant en évidence des différences dans l'implantation de certains baraquements par rapport aux plans de projet. (Fig. 4).

Document 4 :Superposition des anomalies rectangulaires détectées lors de la prospection géophysique et du plan réglementaire d'une base hospital (DAO : M. Mariette, Afrac).

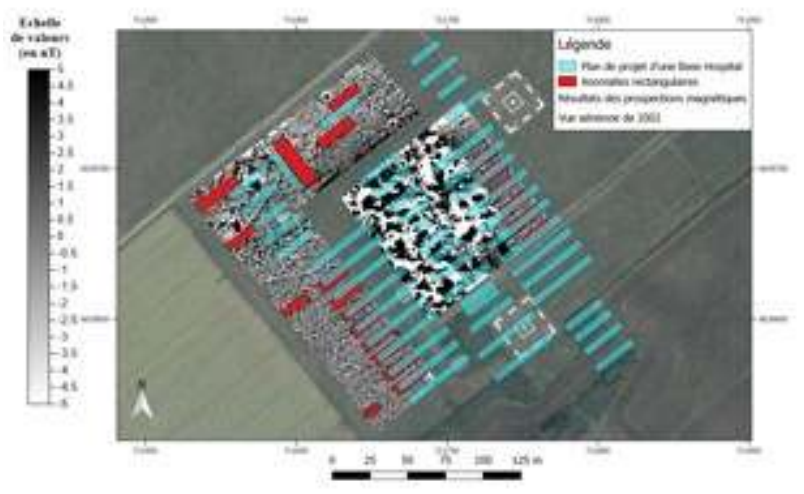

Les techniques géophysiques actuelles permettent d'appréhender de manière concrète les vestiges enfouis et, à l'instar de l'étude des photographies aériennes, offrent un aperçu du potentiel archéologique d'une zone, tout en la préservant d'une opération archéologique intrusive.

\section{Un paysage bouleversé}

\section{A. Une exploitation industrielle des ressources naturelles}

L'utilisation par les américains de bois, de brique et de béton de manière industrielle et massive a sans doute eu un impact sur le paysage français (Coulaud et Perarnau, 2019). Ainsi, on sait que la totalité du bois n'a pu être importée des États-Unis en raison des risques militaires et du défi économique que cela représentait. Le régiment du 20th Engineers(Régiment du Génie, 18543 hommes à la signature de l'armistice) a exploité les forêts de pins du Sud-Ouest de la France, des Vosges et de la forêt d'Argonne à partir du mois d'octobre 1917 (Perarnau, 2016). Ce travail d'exploitation du bois, en bonne entente avec les autorités françaises, impliquait également la construction de scieries entièrement mécanisées et d'un réseau viaire permettant l'acheminement des produits dérivés du bois à travers tout le pays. Ce travail considérable, bien que limité dans l'espace et dans le temps, a eu un impact non négligeable sur l'environnement.

De même, la production locale de béton à partir de gravières situées à proximité des camps (attestées à Beaune et à Mars-sur-Allier), ou encore de briques d'argile locale a 
contribué à modifier le paysage de manière durable. L'activité d'extraction autour du camp américain de Beaune n'a d'ailleurs jamais vraiment cessé par la suite.

\section{B. L'alimentation hydraulique}

Les camps américains nécessitaient un réseau hydraulique complexe afin de permettre

l'alimentation des moteurs à vapeur ainsi que l'accès à une eau potable de qualité. Cette préoccupation des autorités américaines, qui s'explique par les nombreuses épidémies de fièvre typhoïde lors des conflits du XIX ${ }^{\mathrm{e}}$ siècle, incite les ingénieurs américains à redoubler d'efforts pour construire un véritable réseau d'approvisionnement en eau, bien plus complexe que celui préexistant en France en 1917. Ainsi, en juin 1918, on compte 375 projets de construction d'infrastructures hydrauliques, et ce, hors de la zone de front (Tomlin, 1918 inPerarnau, 2016). Il s'agit là autant de travaux de raccordement (canalisations, regards, etc.) que d'infrastructures de traitement et de filtration de l'eau. Ces installations ont considérablement amélioré le réseau français et ont été utilisées encore bien des années après le départ des américains (Perarnau, 2016).

Sur le site de Mars-sur-Allier, la persistance des infrastructures hydrauliques dans le paysage actuel souligne leur importance et leur utilité au sein de ce gigantesque complexe. Ce dernier, d'une capacité d'accueil de 40000 lits (20000 lits permanents sous baraquements et plus de 20000 places sous tentes en cas de crise majeure) est implanté sur 330 hectares et comporte plus de 800 baraques. Son fonctionnement quotidien nécessitait d'énormes quantités d'eau, notamment pour l'alimentation, l'hygiène et la stérilisation des textiles et des instruments médicaux. Le réseau d'adduction et d'assainissement du camp représente près de $205 \mathrm{~km}$ de canalisation en céramique régulièrement entrecoupées de regards en béton (Fig. 5D), facilitant l'entretien des conduites (Mariette, 2017 ; Coulaud, à paraître). L'eau était pompée en amont du camp, dans le cours de l'Allier, via une station de pompage bétonnée (Fig. 5A). Celle-ci était adjointe de quatre pompes rotatives d'une capacité de 25000 gallons à l'heure (soit environ 95000 litres). L'eau était ensuite envoyée via une station relais (Fig. 5B) vers le camp, puis stockée dans un réservoir semi-circulaire en béton de $840 \mathrm{~m}^{3}$, construit en juin 1918 (Mariette, 2017) (Fig. 5E). Un second réservoir de moindre importance vient compléter la capacité de stockage (Coulaud et Tisserand, 2014a ; 2014b). Les eaux usées étaient ensuite évacuées vers un important bassin de décantation bétonné (Fig. $5 \mathrm{~F}$ ) et, après filtration, étaient rejetées dans le cours d'eau voisin. Enfin, un château d'eau en béton (Fig. 5C) fut érigé à la fin du conflit mais ne fut jamais utilisé, même après la signature de l'armistice. C'est à ce jour le vestige le mieux conservé du site. D'une capacité d'environ $380 \mathrm{~m}^{3}$, il présente une plaque métallique arborant l'insigne de l'armée américaine ainsi que plusieurs graffitis mormons traduisant l'origine de ses bâtisseurs : l'état d'Utah (Mariette, 2017; Fig. 5). Sur le site de Mars-sur-Allier, la majeure partie des vestiges encore existants correspondent à des structures hydrauliques.

Document 5 : Vestiges du réseau hydraulique du centre hospitalier de Mars-sur-Allier (Nièvre)

A : Station de pompage sur l'Allier. B : Vestiges de la station relais (M. Mariette, Afrac). C : Château d'eau (A. Coulaud, Inrap). D : Regard en béton et canalisations en céramique après fouille (A. Coulaud, Inrap). E : Vue aérienne du grand réservoir semi-enterré (A. Coulaud, Inrap). F : Vue générale du bassin de décantation (M. Mariette, Afrac). 


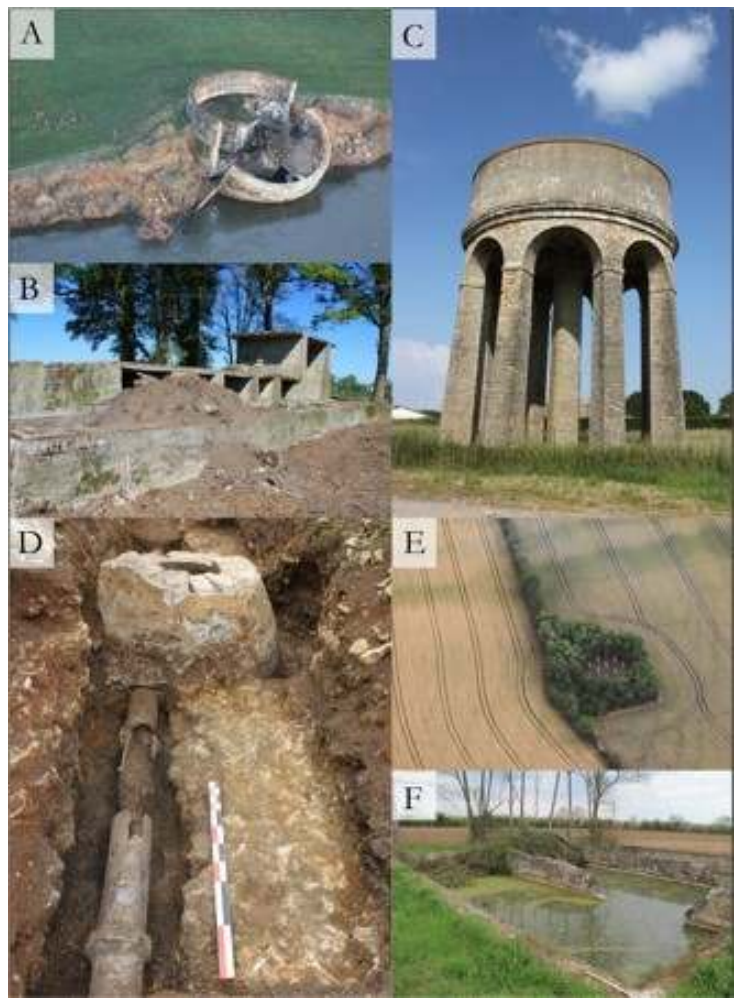

\section{Un paysage révélateur}

Après le départ des troupes américaines, la plupart des sites alors occupés sont totalement abandonnés. Certaines structures sont démantelées par les propriétaires des terrains, agriculteurs ou riverains. Les matériaux, réemployés, servent au développement urbain d'après-guerre. En revanche, les secteurs qui ont été délaissés connaissent un développement naturel de la végétation; et ce, dans un premier temps, autour des vestiges. Sur les photographies aériennes, les traces anthropiques sont mises en valeur par ces espaces végétalisés. Pour les années 1940 à 1970, il est ainsi possible de distinguer ces vestiges, et d'identifier les contours des anciennes constructions. Par ailleurs, en couplant l'étude des photographies aériennes anciennes et la détermination des essences végétales sur le terrain, le développement de la végétation est retraçable. Il en découle une vision concrète de l'implantation et de l'évolution de la flore, mais aussi de l'état de conservation des vestiges.

La présence de végétation peut garantir la bonne conservation des vestiges, si celle-ci est présente de manière suffisamment abondante pour les cacher aux yeux des hommes. C'est notamment le cas de restes maçonnés de baraquements présents en surface que l'on retrouve sur les sites des hôpitaux militaires américains de MesvesBulcy et Mars-sur-Allier (Charrière, 2016 et 2017; Mariette, 2017 et 2018), partiellement ou entièrement conservés sous couvert forestier.

Par ailleurs, la végétation peut aussi constituer un marqueur d'une activité anthropique ancienne, dont les traces matérielles ont aujourd'hui disparu. En effet, certains végétaux ont fossilisé dans le paysage les empreintes d'anciens vestiges. C'est le cas pour le contournement ferroviaire de la ville de Nevers. Ce dernier, construit par les Américains afin d'éviter le passage des trains au sein même de la ville, fut entièrement détruit et démonté entre 1920 et 1924, après le départ des alliés. Le tracé 
de la voie ferrée reste cependant perceptible aujourd'hui. Ainsi, la végétation qui s'y est installée après les travaux de démontage possède des caractéristiques différentes de celle de la végétation alentours. C'est ce différentiel qui met en évidence cette ancienne occupation.

Au sein de l'hôpital de Mars-sur-Allier, une des Base Hospitals sous couvert végétal est caractérisée par des différences nettes de végétation en fonction des secteurs (Mariette, 2018). On retrouve ainsi des essences végétales typiques d'une friche dense sur les zones réservées aux dortoirs et aux annexes (garages, incinérateurs, etc.), tandis que sur les zones dédiées aux bâtiments médicaux (rayons X, morgues, salles d'opérations, pharmacies, etc.), celles-ci correspondent à des espaces de clairière et de prairie. Cette zone étant préservée de toute activité humaine depuis l'abandon du site par les Américains, il est envisageable que cette différence soit liée à une persistance des pollutions anciennes. Ainsi, les activités des bâtiments médicaux ont pu freiner l'implantation de certaines essences ou le développement de certaines autres.

Ces observations, inédites, emmènent les recherches archéologiques contemporaines sur de nouvelles pistes d'études. Elles ouvrent aussi la voie à de nouvelles thématiques de recherche en matière d'environnement et de persistance des pollutions.

Bien que certains de ces sites archéologiques aient été préservés, nombre d'entre eux ont subis les effets de l'urbanisation de l'après-guerre.

31 Il est essentiel de prendre en compte l'urbanisation comme une strate particulière de notre interprétation. En contexte archéologique, elle doit être comprise comme un phénomène de concentration de l'activité humaine autour d'un pôle stratégique, qui soumet l'objet d'étude à un processus de destruction. L'étude de l'urbanisation témoigne ainsi d'un processus accéléré de destruction de grande ampleur du patrimoine archéologique, par la reconversion des terres agricoles en parcelles urbaines, par la disparition d'une sédimentation stratifiée et par la bétonisation des sols.

32 À Beaune (Côte-d'Or), les vestiges d'un camp américain de 1918-1919 sont situés à la périphérie Est de la ville. Les photographies aériennes de 1940 et de 1950 nous ont permis d'associer 277 structures archéologiques à l'activité du camp américain. Or, la même méthode nous permet d'en identifier seulement 74 sur les photographies aériennes de 2017. Ainsi, nous avons cherché en comparant les photographies aériennes prises entre 1950 et 2017, comment ce potentiel archéologique exceptionnel s'est transformé en cible d'une destruction progressive, fruit de l'urbanisation de cette zone périphérique de la ville (Fig. 6).

Document 6 :Zones aménagées entre 1950 et 2017 sur l'emplacement du camp américain de Beaune (Côte-d'Or) (R. Perarnau, Afrac) 


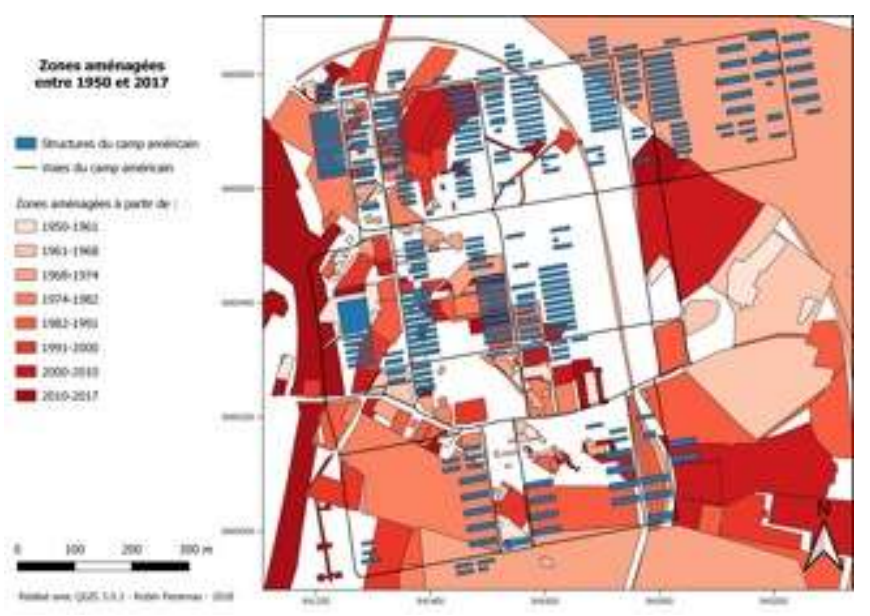

34 Jusqu'en 1968, l'activité autour du camp américain est relativement limitée, on dénombre seulement de petits aménagements, correspondant au développement d'industries déjà présentes sur place, ou encore à la construction de quelques résidences de manière dispersée. Seule l'activité d'extraction de roche à l'Est du camp paraît susceptible de menacer les vestiges.

Entre 1968-1974, la mise en place d'infrastructures viaires accélère la destruction du site. En effet, la construction de l'échangeur autoroutier entre l'A6 et l'A31 impacte 57 structures soit $21 \%$ du camp. Le rail de service construit dans cette même période détruit également une dizaine de structures.

Entre 1974 et 1982, c'est la construction de zones pavillonnaires qui amorce la création d'un véritable quartier résidentiel dont le développement progressif ne cessera jamais véritablement.

À partir de 1982, l'essor de nouvelles zones industrielles et l'extension de zones préexistantes viennent achever de détruire les parties sud et sud-est du camp. Cette tendance, conjointement à la création de zones pavillonnaires, va progressivement détruire le site jusqu'à aujourd'hui. À cela s'ajoute l'impact de la construction des infrastructures à l'ouest du camp, construites à partir des années 2010 (notamment la nouvelle rocade).

L'urbanisation sur le camp américain de Beaune se traduit donc en terme d'activité par le développement de zones industrielles et l'extension de carrières, de grandes infrastructures viaires (autoroute, rail, rocade) et le développement de quartiers résidentiels pavillonnaires en périphérie de la ville.

On voit ainsi très clairement que les parties sud et est du camp ont été détruites par de grands travaux industriels (carrières, usines, etc.) et que les parties nord-est et ouest ont surtout été impactées par les infrastructures viaires. La partie centrale a quant à elle plutôt été détruite par la construction d'une zone résidentielle.

40 Ainsi, sur les 277 structures répertoriées, seules 74 n'ont pas été impactées par un aménagement, soit 27\%. 73\% des vestiges présents en 1950 ont donc été détruits partiellement ou totalement. Si les aménagements entre 1968 et 1982 ont brutalement fait disparaître $44 \%$ des vestiges, la tendance à la destruction est relativement stable et continue depuis 1982.

41 Hormis la destruction des vestiges, l'urbanisation signifie aussi pour l'archéologue d'aujourd'hui un travail de prospection plus difficile en raison de la parcellisation du 
terrain, nécessitant un temps non compressible de prise de contact avec les habitants. Il n'est ainsi pas rare de devoir travailler dans les jardins de propriétés privées. Par ailleurs, la proximité d'habitations avec un site archéologique peut faciliter le pillage et le réemploi des matériaux, effaçant ainsi les traces matérielles.

\section{Conclusion}

La participation des États-Unis dans la Grande Guerre reste un évènement mal connu du grand public. Néanmoins les différentes recherches menées depuis 2014 laissent entrevoir une opération historique majeure. Si l'impact stratégique sur le front reste limité, la quantité de vestiges découverts depuis les débuts de ces recherches vient conforter l'idée d'une logistique très importante dans la vision opérationnelle du conflit par les États-Unis.

Même si à l'issue de la guerre, l'armée française, quoique fortement diminuée, est considéré comme l'armée la plus moderne et la plus aboutie de son temps (Goya, 2004 ; 2018), les États-Unis d'Amérique deviennent une des plus puissantes économies mondiales, soutenue par une armée professionnelle de première importance. Ainsi, la maîtrise de la logistique opérationnelle par l'U.S. Armyva devenir la clé de voute des théâtres d'opérations futurs et ce jusqu'à aujourd'hui. Avec les leçons acquises du premier conflit mondial où l'American Expeditionnary Forces établit les bases de cette logistique de campagne, ce même schéma sera répété pour les opérations américaines de la Seconde guerre mondiale (Débarquements de Normandie, de Provence, de Sicile, opérations du Pacifique), de la Guerre de Corée ou de celles du Vietnam.

Si notre recherche s'est focalisée sur le territoire de la Bourgogne, elle a démontré la diversité des vestiges contemporains et l'importance de la démarche archéologique. L'inventaire réalisé en 2016 souligne que de nombreux sites restent encore à explorer. La diversité des problématiques (choix des matériaux de construction, organisation spatiale des différents sites ou encore analyse des pratiques funéraires) requiert la mise en place d'une méthodologie pluridisciplinaire : recherche archivistique, analyse des photographies aériennes, prospections pédestre et géophysique, étude géochimique et sondages archéologiques.

\section{BIBLIOGRAPHIE}

Charrière G., 2016, Les vestiges des hôpitaux militaires américains en France: une archéologie de la Grande Guerre, Mémoire de Master I AGES, Université de Bourgogne/Franche-Comté, sous la direction de A. Coulaud, Dijon, 53 p. + annexes

Charrière G., 2017, Étude archéologique du réseau hospitalier américain de la Première Guerre mondiale: l'exemple du camp de Mesves dans la Nièvre, Mémoire de Master II AGES, Université de Bourgogne/ Franche-Comté, sous la direction de A. Coulaud, Dijon, 70 p. + annexes 
Coulaud, A., à paraître, Évacuation des eaux, U.S. Army Base Hospital 14. Hospital center (1917-1919), Mars-sur-Allier (Nièvre), Rapport final d'opération, Fouille archéologique programmée 2016

Coulaud A. et Perarnau R. en collaboration avec Charrière G. et Mariette M., 2019, « La baraque militaire dans les hôpitaux américains de la Grande Guerre. Stratégies économiques et choix socio-culturels », Archéopages, n46, « Maisons », pp. 80-85

Coulaud A. et Tisserand N., 2014a, Occupation néolithique et témoignage exceptionnel de la Première Guerre mondiale: l'hôpital américain de Saint-Parize-le-Châtel (Nièvre), volume 1: documentation administrative et résultats, rapport de diagnostic, Inrap, Dijon, $97 \mathrm{p}$.

Coulaud A. et Tisserand N., 2014b, Occupation néolithique et témoignage exceptionnel de la Première Guerre mondiale: l'hôpital américain de Saint-Parize-le-Châtel (Nièvre), volume 2 : figures, rapport de diagnostic, Inrap, Dijon, 86 p.

Coulaud A. et Tisserand N., 2016a, «L'Hospital Center de Mars-sur-Allier dans la Nièvre. Un gigantesque complexe », Archéologia, n548, Dijon, Editions Faton, pp. 44-45

Coulaud A. et Tisserand N., 2016b, « Organisation médicale pendant la Première Guerre mondiale. L'exemple de l'hôpital américain de Saint-Parize-le-Châtel ", Archéopages, 43, "Médecines", Inrap, pp. 68-71

Goya M., 2004, L'invention de la guerre moderne. Du pantalon rouge au char d'assaut. 1871-1918, Editions Texto, $480 \mathrm{p}$.

Goya M., 2018,Les vainqueurs. Comment la France a gagné la Grande Guerre, Editions Taillandier, 352 p.

Mariette M., 2018, Étude géophysique d'un site archéologique contemporaine: l'hôpital américain de la Grande Guerre de Mars-sur-Allier, 1917-1919 (Saint-Parize-le-Châtel, Nièvre),Mémoire de Master II ASA, Université de Bourgogne/Franche-Comté, sous la direction de A. Coulaud et A. Quiquerez, Dijon, 68 p. + annexes

Mariette M., 2017, Approche archéologique d'un site contemporain: l'hôpital militaire américain de Marssur-Allier (1917-1919) (Saint-Parize-le-Châtel, Nièvre), Mémoire de Master I AGES, Université de Bourgogne/Franche-Comté, sous la direction de A. Coulaud, F. Devevey et R. Perarnau, Dijon, 50 p. + annexes

Miquel P., 1983, La Grande Guerre, Paris, Editions Fayard, 663 p.

Perarnau R., 2016, Contribution à l'étude archéologique de la logistique américaine durant la Première Guerre mondiale à travers l'exemple du camp américain de Beaune (1918-1919), Mémoire de Master II AGES, Université de Bourgogne, sous la direction de A. Coulaud, Dijon, 72 p. + annexe

\section{RÉSUMÉS}

En avril 1917, les États-Unis entrent en guerre contre les Empires centraux aux côtés de la Triple Entente. Petite armée professionnelle, l'armée américaine prévoit, à terme, l'envoi de près de 4 millions d'hommes sur le territoire français. Ce défi opérationnel amène l'American Expeditionnary Forces à déployer un impressionnant réseau logistique. Ainsi, afin de subvenir aux besoins $\mathrm{du}$ corps expéditionnaire, l'U.S. Armymet en place plusieurs centaines d'infrastructures logistiques et médicales sur l'ensemble du territoire français : hôpitaux, cliniques vétérinaires, gares régulatrices, camps de stationnement, dépôts de munitions, réseaux ferroviaires, etc. Entre les ports de débarquement de la façade Atlantique et de la Méditerranée, les bases arrière et le front, un réseau vital tentaculaire voit le jour. Parfois loin des zones de 
combat, de vastes complexes marquent toujours le paysage et le territoire français. Depuis plusieurs années, des prospections, des travaux universitaires, des sondages et des fouilles archéologiques s'attèlent à mettre au jour les vestiges de cette opération extraordinaire.

In april 1917, The United States of America enter the Great War against the Triple-Alliance alongside The Triple-Entente. Small professionnal army, the US Army plans to send over timeabout 4 millions soldiers in France. This logistical challenge leads the American Expeditionnary Forces to deploy one of the most impressive logistical grids of all time.Premises of the contemporary operational logistic like the great military operations from the World War II to the present day, the US Army has put in place few hundreds of logistical and medical structures on the whole French territory to support the expeditionary corps: hospitals, veterinary hospitals, regulatory stations, stationing camps, logistical and ammunition warehouses, rail grids,... Between landing ports on the French Atlantic and Mediterranean coasts, support bases and battlefront, a vital sprawling grid see the light of day. Sometimes, away from the battlefields, vast but not well-known facilities still mark the landscape of the French country. For few years, non-intrusive explorations, university research and archaeological surveys try to unearth this huge logistical grid at the rear of the battlefields during a conflict from wich the United States of America get out as the world first economic power.

\section{INDEX}

Mots-clés : American Expeditionnary Forces, logistique, réseau ferroviaire, hôpital, prospections archéologiques, photographies aériennes

Keywords : American Expeditionnary Forces, logistic, rail grid, hospital, archaeological exploration, aerial photographies

\section{AUTEURS}

ALEXANDRE COULAUD

Inrap / Afrac, 842 chemin Saint-Antoine, 97300 Cayenne ; alexandre.coulaud@inrap.fr

\section{MANON MARIETTE}

Afrac, 287 rue des Grands Genêts, 58130 Urzy ; manon.mariette.95@gmail.com

\section{ROBIN PERARNAU}

Afrac, 29 rue des Capucins, 51100 Reims ; perarnau.robin@gmail.com 